\title{
On Narrow Norms and Vague Heuristics: A Reply to Kahneman and Tversky (1996)
}

\author{
Gerd Gigerenzer \\ Max Planck Institute for Psychological Research
}

\begin{abstract}
This reply clarifies what G. Gigerenzer's (e.g., 1991, 1994; Gigerenzer \& Murray, 1987) critique of the heuristics-and-biases approach to statistical reasoning is and is not about. At issue is the imposition of unnecessarily narrow norms of sound reasoning that are used to diagnose so-called cognitive illusions and the continuing reliance on vague heuristics that explain everything and nothing. $D$. Kahneman and A. Tversky (1996) incorrectly asserted that Gigerenzer simply claimed that frequency formats make all cognitive illusions disappear. In contrast, Gigerenzer has proposed and tested models that actually predict when frequency judgments are valid and when they are not. The issue is not whether or not, or how often, cognitive illusions disappear. The focus should be rather the construction of detailed models of cognitive processes that explain when and why they disappear. A postscript responds to Kahneman and Tversky's (1996) postscript.
\end{abstract}

I welcome Kahneman and Tversky's (1996) reply to my critique (e.g., Gigerenzer, 1991, 1994; Gigerenzer \& Murray, 1987) and hope this exchange will encourage a rethinking of research strategies. I emphasize research strategies, rather than specific empirical results or even explanations of those results, because I believe that this debate is fundamentally about what constitutes a good question and a satisfactory answer in psychological research on reasoning. Hence I shall devote the bulk of the very few pages allotted to this reply to setting out how our research strategies differ and how our disagreements stem from these differences. In the remainder, I shall address the key empirical issue: the role of frequency formats.

The main goal of the heuristics-and-biases program has been "to understand the cognitive processes that produce both valid and invalid judgments" (Kahneman \& Tversky, 1996, p. 582). We agree on the goals, but not on the means. It is understandable that when heuristics were first proposed as the underlying cognitive processes in the early 1970 s, they were only loosely characterized. Yet, 25 years and many experiments later, explanatory notions such as representativeness remain vague, undefined, and unspecified with respect both to the antecedent conditions that elicit (or suppress) them and also to the cognitive processes that underlie them. My fear is that in another 25 years researchers will still be stuck with plausible yet nebulous proposals of the same type: that judgments of probability or frequency are sometimes influenced by what is similar (representativeness), comes easily to mind (availability), and comes first (anchoring). The problem with these heuristics is that they at once explain too little and too much. Too little, because we do not know when these heuristics work and how; too much, because, post hoc, one of them can be fitled to almost any experimental result. For example, base-rate neglect is com-

Correspondence concerning this article should be addressed to Gerd Gigerenzer, Center for Adaptive Behavior and Cognition, Max Planck Institute for Psychological Research, Leopoldstrasse 24, 80802 Munich, Germany. Electronic mail may be sent via Internet to giger@ mpipf-muenchen.mpg.de. monly attributed to representativeness. However, the opposite result, overweighting of base rates (conservatism), is as easily "explained" by saying the process is anchoring (on the base rate) and adjustment. Why has there been so little progress in fleshing out the cognitive processes?

I have argued that there are two major obstacles to understanding these cognitive processes. The first is that the norms for evaluating reasoning have been too narrowly drawn, with the consequence that judgments deviating from these norms have been mistakenly interpreted as "cognitive illusions." The second is that vague heuristics have directed attention away from detailed models of cognitive processes and toward posthoc accounts of alleged errors. Let me develop each point in turn.

\section{Narrow Norms}

The first issue on which Kahneman and Tversky and I disagree concerns the question of what counts as sound statistical reasoning. Most practicing statisticians start by investigating the content of a problem, work out a set of assumptions, and, finally, build a statistical model based on these assumptions. The heuristics-and-biases program starts at the opposite end. A convenient statistical principle, such as the conjunction rule or Bayes's rule, is chosen as normative, and some real-world content is filled in afterward, on the assumption that only structure matters. The content of the problem is not analyzed in building a normative model, nor are the specific assumptions people make about the situation.

For instance, consider the Linda problem, in which participants read a description that suggests that Linda is a feminist and are asked "Which is more probable? (a) Linda is a bank teller [ T], or (b) Linda is a bank teller and active in the feminist movement [T\&F]." Tversky and Kahneman's (1983) norm of sound reasoning here is the conjunction rule, and only the conjunction rule, which leads them to conclude that answering $T \& F$ is a fallacy. This norm is narrow in two senses. First, probability theory is imposed as a norm for a single event (whether Linda is a bank teller); this would be considered misguided by 
those statisticians who hold that probability theory is about repeated events (Gigerenzer, 1994). Second, the norm is applied in a content-blind way; that is, with the assumption that judgments about what counts as sound reasoning may ignore content and context (Gigerenzer \& Murray, 1987, chap. 5).

\section{Single-Event Probabilities}

Karl Popper (1990) has recounted that when he learned that Carnap treated the probability of a hypothesis as a mathematical probability, he "felt as a father must feel whose son has joined the Moonies" (p. 5). I share the worries of those statisticians, philosophers, and psychologists who caution that the laws of probability do not apply to all kinds of statements about singular events, but apply only in well-defined circumstances (Gigerenzer et al., 1989). Kahneman and Tversky, in contrast, have subscribed to a controversial doctrine that indiscriminately evaluates all statements about single events by the laws of probability. I do not object to their philosophy; everyone is free to have one. But I do object to imposing it as a norm of sound reasoning on the participants in psychological experiments and as a criterion by which to diagnose cognitive illusions. For instance, the conjunction rule applied to a single event is not a general norm for sound reasoning. This is a critical point of disagreement.

Kahneman and Tversky (1996) defended their narrow norm by pointing out that most people would reason according to the conjunction rule when asked whether a person is more likely to die within a week than within a year. However, from the fact that humans occasionally reason in accordance with the conjunction rule, it does not follow that we always ought to; that is, it does not follow that the conjunction rule is a universally applicable norm of sound reasoning. One can easily create a context, such as a patient already on the verge of dying, that would cause a sensible person to answer that this patient is more likely to die within a week (inferring that the question is next week versus the rest of the year, because the question makes little sense otherwise). In the same fashion, the Linda problem creates a context (the description of Linda) that makes it perfectly valid not to conform to the conjunction rule. Moreover, Kahneman and Tversky argued that the reluctance of statisticians to make probability theory the norm of all single events "is not generally shared by the public" (p. 585). If this was meant to shift the burden of justification for their norms from the normative theory of probability to the intuitions of ordinary people, it is exceedingly puzzling. How can people's intuitions be called upon to substitute for the standards of statisticians, in order to prove that people's intuitions systematically violate the normative theory of probability? By linking arms with John Q. Public, Kahneman and Tversky may find themselves closer than they like to the position of Cohen (1981), who proposed that the intuitions of untutored people serve as the final criterion of rationality.

\section{Content-Blind Norms}

Kahneman and Tversky (1996) have created the impression that my critique of narrow norms (Gigerenzer, 1991, 1994; Gigerenzer \& Murray, 1987) concerned only the issue of single events and that it thus applies only to a few of their phenomena. ${ }^{1}$
They did not address my critique of their practice of imposing a statistical principle as a norm without examining content (e.g., Gigerenzer \& Murray, 1987, pp. 162-174). Many demonstrations of biases in probabilistic reasoning are founded on this practice, as pointed out, for instance, by Einhorn and Hogarth (1981) and Lopes and Oden (1991). Content-blind norms are appropriate for textbook problems in probability theory, where the content is only decorative, but they are not appropriate either for evaluating human judgment or as a research tool to uncover the underlying processes.

For instance, on Kahneman and Tversky's (1996) view of sound reasoning, the content of the Linda problem is irrelevant; one does not even need to read the description of Linda. All that counts are the terms probable and and, which the conjunction rule interprets in terms of mathematical probability and logical AND, respectively. In contrast, I believe that sound reasoning begins by investigating the content of a problem to infer what terms such as probable mean. The meaning of probable is not reducible to the conjunction rule (Hertwig \& Gigerenzer, 1995). For instance, the Oxford English Dictionary (1971, pp. 1400-1401) lists "plausible," "having an appearance of truth," and "that may in view of present evidence be reasonably expected to happen," among others. These legitimate meanings in natural language have little if anything to do with mathematical probability. Similarly, the meaning of and in natural language rarely matches that of logical AND. The phrase T\&F can be understood as the conditional "If Linda is a bank teller, then she is active in the feminist movement." Note that this interpretation would not concern and therefore could not violate the conjunction rule. Recent studies using paraphrasing and protocols suggest that participants draw a variety of semantic inferences to make sense of the Linda problem: Some 10 to $20 \%$ seem to infer that and should be read as a conditional, and some 20 to $50 \%$ seem to infer that the alternative "Linda is a bank teller" implies that she is not active in the feminist movement (e.g., Dulany \& Hilton, 1991; Hertwig \& Gigerenzer, 1995; Macdonald \& Gilhooly, 1990; Tversky \& Kahneman, 1983). These semantic inferences can lead to choosing $T$ rather than T\&F. Semantic inferences-how one infers the meaning of polysemous terms such as probable from the content of a sentence (or the broader context of communication ) in practically no time-are extraordinarily intelligent processes. They are not reasoning fallacies. No computer program, to say nothing of the conjunction rule, has yet mastered this form of intelligence. Significant cognitive processes such as these will be overlooked and even misclassified as "cognitive illusions" by content-blind norms.

\section{Vague Heuristics}

The heuristics in the heuristics-and-biases program are too vague to count as explanations. They are labels with the virtue of Rorschach inkblots: A researcher can read into them what he or she wishes. The reluctance to specify precise and falsifiable process models, to clarify the antecedent conditions that elicit various heuristics, and to work out the relationship between

\footnotetext{
'Incidentally, Kahneman and Tversky (1996) claimed that in their 1974 Science article, only 2 of 12 biases concern single-event probabilities. I found a total of 13 biases, 5 of which involved some example of single-event probabilities.
} 
heuristics have been repeatedly pointed out (e.g., Einhorn \& Hogarth, 1981; Shanteau, 1989; Wallsten, 1983). The two major surrogates for modeling cognitive processes have been (a) one-word-labels such as representativeness that seem to be traded as explanations and (b) explanation by redescription. Redescription, for instance, is extensively used in Kahneman and Tversky's ( 1996) reply. Recall Molière's parody of the Aristotelian doctrine of substantial forms: Why does opium make you sleepy? Because of its dormative properties. Why does a frequency representation cause more correct answers? Because "the correct answer is made transparent" ( p. 586). Why is that? Because of "a salient cue that makes the correct answer obvious" (p. 586), or because it "sometimes makes available strong extensional cues" (p. 589). Researchers are no closer to understanding which cues are more "salient" than others, much less the underlying process that makes them so.

I believe that there is a connection between narrow norms and the absence of process models. Two research strategies on the widely known cab problem illustrate this. Birnbaum (1983) proceeded as a practicing statistician might: first investigating the content of the problem, making various assumptions about processes in eyewitness testimony (e.g., a signal detection model in which the witness tries to minimize some error function), and finally proposing various models of the underlying cognitive processes. As far as I can see, his work has met with little or no interest within the heuristics-and-biases program. Tversky and Kahneman's (1982) analysis of the same problem, in contrast, did not start with the content, but with a statistical rule, Bayes's theorem. They assumed that there is only one "correct answer" (p. 157), to be determined mechanically by plugging in values and computing outcomes. The content of the problem, the various assumptions a problem solver can make about the situation, and how a statistical model is built from these were not parts of their idea of valid statistical reasoning. Blue cabs might as well be the white swans of logical exercises. In the tradition of heuristics and biases, an avalanche of studies have been conducted on the cab problem; as far as I can see, not one model of cognitive processes has emerged.

Several researchers have tried to overcome this state of theoretical minimalism by proposing specific models of cognitive processes (e.g., Birnbaum, 1983; Smith \& Osherson, 1989), including satisficing algorithms that predict individual inferences and make surprising and falsifiable predictions, such as conditions under which less knowledge leads to more accurate inferences (Gigerenzer \& Goldstein, in press). Kahneman and Tversky (1996), however, have continued to defend undefined concepts: Representativeness "can be assessed experimentally; hence it need not be defined a priori" (p. 585). It is hard to see how this reluctance to propose precise models fits with the stated goal of specifying the underlying cognitive processes. One can certainly start by relying only on numerical assessments, but at some point the properties of what is measured need to be more sharply characterized.

I have always found it difficult to understand why Kahneman and Tversky have persisted in one-word explanations such as representativeness, which they still characterize as "an assessment of the degree of correspondence between a sample and a population, an instance and a category, an act and an actor, or more generally between an outcome and a model. The model may refer to a person, a coin, or the world economy, and the respective outcomes could be marital status, a sequence of heads and tails, or the current price of gold" (1996, p. 584). If you are puzzled as to why they feel " [p] erhaps the most serious misinterpretation of our position . . . [is] the characterization of judgmental heuristics as 'independent of context and content' . . . and insensitive to problem representation" (p. 583), then I join you in this sentiment. There is no specification of the cognitive process labeled representativeness (or those corresponding to other heuristics), and a fortiori, no theory of how this process relates to any specific content, context, or representation of numerical information. Kahneman and Tversky pointed to the framing effect, for which specific and interesting models do exist. But my critique (e.g., Gigerenzer, 1991, 1994; Gigerenzer \& Murray, 1987) is of judgmental heuristics, which lack theoretical specification.

The reader may now understand why Kahneman and Tversky ( 1996) and I construe this debate at different levels. Kahneman and Tversky centered on norms and were anxious to prove that judgment often deviates from those norms. I am concerned with understanding the processes and do not believe that counting studies in which people do or do not conform to norms leads to much. If one knows the process, one can design any number of studies wherein people will or will not do well. With this difference in mind, I now look at the principal empirical issue that divides me from Kahneman and Tversky.

\section{The Issue Is Not Whether or Not "Cognitive Illusions" Disappear, But Why}

What Kahneman and Tversky (1996) have presented as the strongest point in defense of their program has figured prominently in the abstract: "Contrary to his central empirical claim, judgments of frequency-not only subjective probabilitiesare susceptible to large and systematic biases" (p. 582). Reader beware.

My colleagues and I have proposed models of cognitive processes that predict when frequency and probability judgments are valid and when they are invalid according to certain norms, and that also explain why. For instance, the theory of probabilistic mental models specifies conditions under which frequency judgments exhibit underestimation of actual frequencies and, more generally, conditions that make "overconfidence bias" appear, disappear, and invert (Gigerenzer, 1993; Gigerenzer, Hoffrage, \& Kleinbölting, 1991). In other work we have shown that when numerical information about base rates, hit rates, and false-alarm rates is represented by absolute frequencies ("natural sampling") rather than by relative frequencies or probabilities, then the proportion of judgments conforming to Bayesian principles increased by about a factor of three. The reason absolute frequencies make a difference and relative frequencies do not is that cognitive algorithms designed to do Bayesian reasoning with absolute frequencies (natural sampling) involve fewer steps of mental computation (Gigerenzer \& Hoffrage, 1995).

Kahneman and Tversky (1996; who referenced these three articles) were mute about these models that specify when frequency judgments are valid and when not. In a curious distortion, they insinuated throughout their reply that 1 simply claimed that all frequency judgments are valid. For instance, "the major empirical point made by Gigerenzer is that the use 
of frequency reliably makes cognitive illusions 'disappear" " (p. 583); and "Contrary to Gigerenzer's unqualified claim, the replacement of subjective probability judgments by estimates of relative frequency ... [does] not provide a panacea against base-rate neglect" ( p. 584). No citations were given.

What Kahneman and Tversky (1996) perceived as the major threat to the "reality of cognitive illusions" is the result that a slight change in the setup-using particular frequency representations or frequency judgments-makes cognitive illusions largely disappear. But this perception misses the main point, because it is concerned solely with deviations from norms and ignores the theories of why these occur. (Note that their notions of "inside" and "outside" views cannot explain when frequency judgments have an effect and when not, nor why some frequency representations facilitate Bayesian reasoning and others do not.)

Hence, Kahneman and Tversky (1996) believe that the appropriate reply is to show that frequency judgments can also fail. There is no doubt about the latter; as mentioned before, my colleagues and 1 (Gigerenzer, 1993; Gigerenzer \& Hoffrage, 1995; Gigerenzer, Hoffrage, \& Kleinbölting, 1991) actually have proposed and tested theories about why and when this is the case. Nonetheless, under appropriate conditions, the effect of frequency is stronger than that of any other "debiasing" method. Overconfidence bias (overestimation) completely disappears (Gigerenzer et al., 1991); the conjunction fallacy in the Linda problem is reduced from about $85 \%$ to $20 \%$ and less (Fiedler, 1988; Hertwig \& Gigerenzer, 1995; see also Tversky \& Kahneman, 1983); and people reason the Bayesian way in about $50 \%$ (Gigerenzer \& Hoffrage, 1995) or 75\% (Cosmides \& Tooby, 1996) of the cases.

Kahneman and Tversky's (1996) response was simultaneously to downplay the effect of frequency and to claim they found it first. "Gigerenzer has essentially ignored our discovery of the effect of frequency" (p. 586). It is correct that they demonstrated the effect on conjunction violations first (but not for overconfidence bias and the base-rate fallacy). Their accusation, however, is out of place, as are most others in their reply. I referenced their demonstration in every one of the articles they cited (e.g., Gigerenzer, 1991, p. 92; 1993, p. 294; 1994, p. 144; Gigerenzer et al., 1991, p. 525). It might be added that Tversky and Kahneman (1983) themselves paid little attention to this result, which was not mentioned once in some four pages of discussion.

Kahneman and Tversky (1996) reported various results to play down what they believe is at stake, the effect of frequency. In no case was there an attempt to figure out the cognitive processes involved. First, they pointed out that even if one replaced the polysemous term probable by asking participants to bet on the outcome of a dice game (Tversky \& Kahneman, 1983), conjunction violations were fairly stable. However, Fiedler (1988) has shown that with frequency judgments, conjunction violations in the dice problem also largely disappear. Second, they cited their seven-letters study to show that even with frequency judgments, people commit the conjunction fallacy. Their reports on this study are somewhat confusing-Kahneman and Tversky ( $1996 ;$ p. 586) described it as a within-subjects design, but Tversky and Koehler $(1994$, p. 547) reported the same study as a between-subjects design; the original article ( Tversky \& Kahneman, 1983) offers no information on this issue. If
Kahneman and Tversky are right, then the effect depends crucially on presenting the two alternatives to a participant at different times, that is, with a number (unspecified in their reports) of other tasks between the alternatives. This does not seem to be a violation of internal consistency, which I take to be the point of the conjunction fallacy. Similarly, the betweensubjects version of the Linda problem is not a violation of internal consistency, because the effect depends on not presenting the two alternatives to the same subject. The effect reported is nevertheless interesting and consistent with Macdonald and Gilhooly's ( 1990) result that T was judged less likely in the absence of $\mathrm{T} \& \mathrm{~F}$ than in its presence (see Hertwig \& Gigerenzer, 1995).

Concerning base-rate neglect, Kahneman and Tversky (1996; p. 584) created the impression that there is little evidence that certain types of frequency formats improve Bayesian reasoning. They do not mention that there is considerable evidence (e.g., Gigerenzer \& Hoffrage, 1995) and back their disclaimer principally with a disease-classification study by Gluck and Bower (1988), which they summarized thus: "subjects" judgments of the relative frequency . . . were determined entirely by the diagnosticity of the symptom, with no regard for the base-rate frequencies of the diseases" (p. 584, italics added). To set the record straight, Gluck and Bower said their results were consistent with the idea that "base-rate information is not ignored, only underused" (p. 235). Furthermore, their study was replicated and elaborated on by Shanks (1991), who concluded that "we have no conclusive evidence for the claim . . . that systematic base-rate neglect occurs in this type of situation" (p. 153).

Adding up studies in which base-rate neglect appears or disappears will lead us nowhere. Progress can be made only when we can design precise models that predict when base rates are used, when not, and why. Both Gluck and Bower (1988) and Shanks ( 1991 ) have designed connectionist models that address base-rate use, and I consider this a step in the right direction.

\section{Conclusion}

There is no question that Kahneman and Tversky's heuristics-and-biases program has had a tremendous stimulating effect on research. But the sheer proliferation of studies is not always identical to progress. An ever-larger collection of empirical results, especially results that seem to vary from study to study in apparently mysterious ways, can be more confusing than clarifying. If the psychology of judgment ultimately aims at an understanding of how people reason under a bewildering variety of circumstances, then descriptions, however meticulous and thorough, will not suffice. In place of plausible heuristics that explain everything and nothing-not even the conditions that trigger one heuristic rather than another-we will need models that make surprising (and falsifiable) predictions and that reveal the mental processes that explain both valid and invalid judgment.

\section{References}

Birnbaum, M. H. (1983). Base rates in Bayesian inference: Signal detection analysis of the cab problem. American Journal of Psychology, $96,85-94$. 
Cohen, L. J. ( 1981). Can human irrationality be experimentally demonstrated? The Behavioral and Brain Sciences, 4, 317-370.

The compact edition of the Oxford English dictionary. (1971). Oxford, England: Oxford University Press.

Cosmides, L., \& Tooby, J. (1996). Are humans good intuitive statisticians after all? Rethinking some conclusions from the literature on judgment under uncertainty. Cognition, 58, 1-73.

Dulany, D. E., \& Hilton, D. J. (1991). Conversational implicature, conscious representation and the conjunction fallacy. Social Cognition, 9.85-110.

Einhorn, H. J., \& Hogarth, R. M. (1981). Behavioral decision theory: Processes of judgment and choice. Annual Review of Psychology, 32, 53-88.

Fielder, K. (1988). The dependence of the conjunction fallacy on subtle linguistic factors. Psychological Research, 50, 123-129.

Gigerenzer, G. (1991). How to make cognitive illusions disappear: Beyond "heuristics and biases." In W. Stroebe \& M. Hewstone (Eds.), European Review of Social Psychology (Vol. 2, pp. 83-115). Chichester, England: Wiley.

Gigerenzer, G. ( 1993). The bounded rationality of probabilistic mental models. In K. 1. Manktelow \& D. E. Over (Eds.), Rationality (pp. 284-313). London: Routledge.

Gigerenzer, G. (1994). Why the distinction between single-event probabilities and frequencies is relevant for psychology and vice versa. In G. Wright \& P. Ayton (Eds.), Subjective probability (pp. 129-162). New York: Wiley.

Gigerenzer, G., \& Goldstein, D. G. (in press). Reasoning the fast and frugal way: Models of bounded rationality. Psychological Review.

Gigerenzer, G., \& Hoffrage, U. (1995). How to improve Bayesian reasoning without instruction: Frequency formats. Psychological Review; 102, 684-704.

Gigerenzer, G., Hoffrage, U., \& Kleinbölting, H. (1991). Probabilistic mental models: A Brunswikian theory of confidence. Psychological Review, 98, 506-528.

Gigerenzer, G., \& Murray, D. J. (1987). Cognition as intuitive statistics. Hillsdale, NJ: Erlbaum.

Gigerenzer, G., Swijtink, Z., Porter, T., Daston, L., Beatty, J., \& Krüger, L. (1989). The empire of chance: How probability changed science and everyday life. Cambridge, England: Cambridge University Press. Gluck, M. A.. \& Bower, G. H. (1988). From conditioning to category learning: An adaptive network model. Journal of Experimental $P_{s y-}$ chology: General, 117, 227-247.

Hertwig, R., \& Gigerenzer, G. ( 1995). The "conjunction fallacy" revisited. Manuscript submitted for publication.

Kahneman, D., \& Tversky, A. (1996). On the reality of cognitive illusions: A reply to Gigerenzer's critique. Psychological Review, 103. 582-591.

Lopes, L. L., \& Oden, G. C. (1991). The rationality of intelligence. In E. Eells \& T, Maruszewski (Eds.), Rationality and reasoning: Essays in honor of L. J. Cohen (pp. 199-223). Amsterdam: Rodopi.

Macdonald, R. R., \& Gilhooly, K. J. ( 1990 ). More about Linda or conjunctions in context. European Journal of Cognitive Psychology, 2, 57-70.

Popper, K. R. (1990), A world of propensities. Bristol, England: Thoemmes.

Shanks, D. (1991). A connectionist account of base-rate biases in categorization. Connection Science, 3, 143-162.

Shanteau, J. (1989). Cognitive heuristics and biases in behavioral auditing: Review, comments, and observations. Accounting Organizations and Society, 14, 165-177.

Smith, E. E., \& Osherson, D. N. ( 1989 ). Similarity and decision making. In S. Vosniadou \& A. Ortony (Eds.), Similarity and analogical reasoning (pp. 60-75). Cambridge, England: Cambridge University Press.

Tversky, A., \& Kahneman, D. ( 1982). Evidential impact of base rates. In D. Kahneman, P. Slovic, \& A. Tversky (Eds.), Judgment under uncertainty: Heuristics and biases (pp. 153-160). Cambridge, England: Cambridge University Press.

Tversky, A., \& Kahneman, D. (1983). Extensional versus intuitive reasoning: The conjunction fallacy in probability judgment. Psychological Review, 90, 293-315.

Tversky, A., \& Koehler, D. J. (1994). Support theory: A nonextensional representation of subjective probability. Psychological Review. 101, 547-567.

Wallsten, T. S. (1983). The theoretical status of judgmental heuristics. In R. W. Scholz (Ed.), Decision making under uncertainty (pp. 2139). Amsterdam: Elsevier.

Received September 8, 1995

Revision received January 5, 1996

Accepted January 5, 1996

\section{Postscript}

Controversies can be healthy opportunities for pause and rethought. Let me conclude this exchange by remarking on two issues in Kahneman and Tversky's (1996) postscript. Concerning the "conjunction effect" (and I do appreciate their shift in terminology from fallacy to effect), Kahneman and Tversky implied that my case rests solely on the Linda problem. This is incorrect; I have repeatedly mentioned work that showed that, for a half dozen problems other than Linda, frequency formats made the conjunction effect largely disappear. The same goes, $m u-$ tatis mutandis, for the other objections I have raised to the heuristics-andbiases program, all of which are based on multiple empirical examples.

However, the important issue that divides us is research strategy. Kah- neman and Tversky's (1996) postscript ended with an appeal for "building on the notions of representativeness, availability, and anchoring" (p. 591). In the same breath, they denied the need for constructing process models and specifying the conditions under which different heuristics work. What sort of "building" can occur without first daring to be precise? As I see it, there are two ways in which a theory can fail: by being wrong or by being indeterminate. The latter may be worse for scientific progress, because indeterminate theories resist attempts to prove, disprove, or even improve them. Twenty-five years ago, extending on Ward Edward's work, Kahneman and Tversky opened up a fertile field. Now it is time to plant theories. 